\title{
Scaling of transient wave-soil interaction problems
}

\author{
Heng Xiao ${ }^{1, *, \dagger}$, Yin Lu Young ${ }^{2}$ and Jean H. Prévost ${ }^{1}$ \\ ${ }^{1}$ Department of Civil and Environmental Engineering, Princeton University, Princeton, NJ 08544, U.S.A. \\ ${ }^{2}$ Department of Naval Architecture and Marine Engineering, University of Michigan, Ann Arbor, MI 48109, U.S.A.
}

\begin{abstract}
SUMMARY
Within the framework of compressible multi-phase flow through deformable porous media, wave-soil interactions in the near-shore region during wave runup and drawdown are modeled. Critical non-dimensional parameters governing the interaction processes are identified. Within the context of wave basin and centrifuge wave tank facilities, we propose scaling relations for the experimental investigations of the transient and steady-state responses of wave-soil systems. Numerical simulations are conducted to illustrate and confirm the theoretical and scaling analyses. Based on the simulations results, the implications on the design of experiments and interpretation of results are discussed. Copyright (C) 2009 John Wiley \& Sons, Ltd.
\end{abstract}

Received 27 July 2009; Accepted 14 August 2009

KEY WORDS: experimental scaling; tsunami; storm surge; wave-soil interaction

\section{INTRODUCTION}

Wave-induced seabed responses near the shoreline are of significant practical and theoretical interests. Wave impact due to tsunamis and storm surges can impair coastal infrastructures, cause erosion and soil failures, and may lead to serious geological hazards. During wave runup and drawdown along the coastal slopes, critical physical processes involving wave-soil interactions include: (i) sediment transport and bed profile changes; (ii) soil matrix consolidation and pore fluids pressure diffusion; and (iii) interstitial multi-phase flows through deformable porous media. Processes (ii) and (iii) are termed collectively as 'bed response' hereafter and are the focus of this paper. Wave-induced bed erosion and sediment transport are not considered. For simplicity, the soil bed is assumed to be composed of noncohesive sand particles.

\footnotetext{
*Correspondence to: Heng Xiao, Department of Civil and Environmental Engineering, Princeton University, Princeton, NJ 08544, U.S.A.

†E-mail: xiao@princeton.edu

Contract/grant sponsor: National Science Foundation; contract/grant numbers: 0649155, 0530759
}

Copyright (C) 2009 John Wiley \& Sons, Ltd. 
The bed response involves the coupling of solid skeleton (matrix of sand particles) deformation and interstitial multi-phase fluid flows. This coupled process can be modeled within the framework of porous media theory, where the constituents (sand grains, water, and air) are assumed to be individual continua, all interpenetrating each other and occupying the whole domain, each being regarded as a phase [1-3]. Numerical simulations in porous media framework have been conducted for saturated flows $[4,5]$, where the interstitial pores are occupied by one fluid, and more recently for unsaturated flows [6], where two or more fluids jointly occupy the pores.

Laboratory model-scale experiments have been conducted in wave basins to study the near-shore transient wave-induced sediment transport mechanisms, bed profile changes, and dynamic bed responses $[7,8]$. The wave propagation and transformation processes are modeled by preserving the Froude number. However, it is difficult to scale the bed materials because scaling down the diameters of noncohesive particles may lead to cohesive particles. This would in turn yield different stress-strain relations and soil failure mechanisms (slope failure and/or liquefaction). In addition, it is very expensive to use large volumes of man-made materials in large-scale laboratory studies.

Another difficulty for experimental studies of soil failure mechanisms in wave basins is the dependence of the material moduli on the confining stress, which leads to nonlinear stress-strain behaviors. This difficulty can be overcome via the use of centrifuge facilities, with specially designed wave makers inside a bucket that can be operated in flight. By choosing the acceleration to be $\lambda g$ (where $\lambda$ is the length scale ratio between the prototype and the model for a geometrically similar model) and using the same sand as in the prototype, the model-scale experiment can reproduce the prototype stresses if the spatial and temporal variations of the applied surface wave loadings are scaled properly.

In the past few decades, geotechnical centrifuge facilities have been used to study stress wave propagation in soils [9], soil-structure interactions, explosion of dynamite in soils [10], among other phenomena. Centrifuges have become a standard geotechnical testing equipment for research and education. Wave making inside a centrifuge was recently attempted to study wave-induced instability of the sediment bed and related liquefaction and sedimentation phenomena $[11,12]$. However, these experimental studies were limited to regular waves over a flat, fully saturated sand bed, so that the effects of wave runup and drawdown over coastal regions were not considered. Moreover, scaling issues of the transient and steady-state behavior of wave-soil interaction problems have not yet been fully explored, particularly for unsaturated soils.

This study aims at: (i) developing proper scaling relations for the experimental investigation of the transient wave-soil interactions in the context of $1 g$ wave basin and $\lambda g$ centrifuge wave tank facilities (referred to as wave basins and centrifuges, respectively, hereafter) and (ii) investigating the consequences of the inability to satisfy all similitude requirements and the resulting implications on the interpretation of experimental results. The focus is to obtain the proper scaling relations to investigate the transient soil responses caused by extreme wave runup and drawdown. Key non-dimensional numbers governing the wave-soil interaction processes are studied systematically via numerical simulations within the framework of compressible multi-phase flow in deformable porous media.

The paper is organized as follows. Section 2 briefly summarizes the mathematical formulation of the bed responses under external wave loading, as well as the derivations and analyses of the key non-dimensional parameters. Section 3 presents the results of scaling analysis for wave basin and centrifuge wave-making facilities, which are validated by the numerical examples shown in Section 4. Other relevant factors and the recommendation are discussed in Section 5. Finally, Section 6 concludes the paper. 


\section{MATHEMATICAL FORMULATION}

The sand bed is modeled within the framework of poromechanics theory with water, air, and solid skeleton jointly occupying the whole domain, forming an unsaturated medium. Attention is focused on 'quasistatic' situations when the dynamic terms can be neglected. The dynamical stress wave propagation, viz, inertial effects, in the skeleton is ignored in this quasistatic analysis because its time scale is much smaller than those of the other processes of interest in this study. The following equations describe the conservations of momentum, total fluid mass, and species mass, respectively $[2,3]$ :

$$
\begin{array}{r}
\nabla \cdot \boldsymbol{\sigma}+\left[(1-\phi) \rho_{\mathrm{s}}+\phi \rho_{\mathrm{f}}\right] \mathbf{b}=0 \\
\phi \frac{\mathrm{d} \rho_{\mathrm{f}}}{\mathrm{d} t}+\nabla \cdot \mathbf{q}+\rho_{\mathrm{f}} \frac{\mathrm{d} \phi}{\mathrm{d} t}=0 \\
\phi \frac{\mathrm{d}\left(\rho_{\alpha} S_{\alpha}\right)}{\mathrm{d} t}+\nabla \cdot\left(\rho_{\alpha} \overline{\mathbf{q}}_{\alpha}\right)+\rho_{\alpha} S_{\alpha} \frac{\mathrm{d} \phi}{\mathrm{d} t}=0
\end{array}
$$

with

$$
\begin{aligned}
\frac{\mathrm{d} \phi}{\mathrm{d} t} & =b \nabla \cdot \mathbf{v}^{\mathrm{s}}+\frac{1}{N} \frac{\mathrm{d} p_{\mathrm{f}}}{\mathrm{d} t} \\
\rho_{\mathrm{f}} & =\sum_{\alpha=1}^{n_{p}} \rho_{\alpha} S_{\alpha}
\end{aligned}
$$

where Biot's coefficient $b$ and modulus $N$ are defined as [3]

$$
\begin{gathered}
\frac{1}{N}=\frac{b-\phi_{0}}{K_{\mathrm{s}}} \\
b=1-\frac{K}{K_{\mathrm{s}}}
\end{gathered}
$$

where $\sigma$ is the total stress tensor of the mixture; $\phi$ is the Lagrangian porosity of the soil; $\rho_{\mathrm{s}}$ and $\rho_{\mathrm{f}}$ is the solid and fluid density; $\mathbf{b}$ is the body force (gravity in this study); $\boldsymbol{q}$ is the total fluid mass flux; $\alpha$ is the index of phases (air and water); $S_{\alpha}$ is the saturation of phase $\alpha ; \overline{\boldsymbol{q}}_{\alpha}$ is the volume flux of phase $\alpha ; \mathbf{v}^{\mathrm{s}}$ the solid velocity; $p_{\mathrm{f}}$ the pore fluid pressure; $n_{p}$ the number of fluid phases $\left(n_{p}=2\right.$ here); $K$ and $K_{\mathrm{s}}$ are the bulk moduli of the solid matrix and of the grains, respectively; $\phi_{0}$ is the initial porosity.

The effective solid stresses $\left(\boldsymbol{\sigma}^{\mathrm{s}}\right)$ and solid velocities $\left(\mathbf{v}^{\mathrm{s}}\right)$ are expressed in terms of solid displacement field $\mathbf{u}^{\mathrm{s}}$ as follows:

$$
\begin{aligned}
& \mathbf{v}^{\mathrm{s}}=\frac{\partial \mathbf{u}^{\mathrm{s}}}{\partial t} \quad \text { and } \quad \boldsymbol{\sigma}=\boldsymbol{\sigma}^{\mathrm{s}}-b p_{\mathrm{f}} \boldsymbol{\delta} \quad \text { with } \\
& \boldsymbol{\sigma}^{\mathrm{s}}=\mathbf{C}: \boldsymbol{\varepsilon}^{\mathrm{s}} \quad \text { and } \quad \boldsymbol{\varepsilon}^{\mathrm{s}}=\nabla_{()} \mathbf{u}^{\mathrm{s}}
\end{aligned}
$$

where $\mathbf{C}$ is a fourth-order constitutive tensor; the symbol ' $:$ ' denotes the contraction product of two tensors; $\boldsymbol{\varepsilon}^{\mathrm{s}}$ is the strain of the skeleton; $\nabla_{()} \mathbf{u}^{\mathrm{s}}=\left(\nabla \mathbf{u}^{\mathrm{s}}+\mathbf{u}^{\mathrm{s}} \nabla\right) / 2$ is the symmetric part of the displacement gradient tensor; $\boldsymbol{\delta}$ is the second-order unit tensor. 
In Equation (2), the term $\mathrm{d} \rho_{\mathrm{f}} / \mathrm{d} t$ is approximated as

$$
\frac{\mathrm{d} \rho_{\mathrm{f}}}{\mathrm{d} t}=\frac{\mathrm{d}}{\mathrm{d} t}\left[\sum_{\alpha=1}^{n_{p}} \rho_{\alpha} S_{\alpha}\right]=\sum_{\alpha=1}^{n_{p}} \rho_{\alpha} S_{\alpha} C_{\alpha} \frac{\mathrm{d} p_{\alpha}}{\mathrm{d} t}
$$

where $p_{\alpha}$ is the phase pressure; $C_{\alpha}$ is the compressibility of fluid phase $\alpha$. The total mass flux $\mathbf{q}$ is modeled according to Darcy's law

$$
\mathbf{q}=\sum_{\alpha=1}^{n_{p}} \rho_{\alpha} \overline{\mathbf{q}}_{\alpha}=-\left(\sum_{\alpha=1}^{n_{p}} \rho_{\alpha} k_{\alpha}\right) \mathbf{k} \cdot[\nabla P-\bar{\rho} \mathbf{b}]
$$

where $\overline{\mathbf{q}}_{\alpha}$ is the volume flux of phase $\alpha ; \mathbf{k}$ is the intrinsic permeability tensor of the soil skeleton (for isotropic material, it is replaced with a scalar $k ; P$ is the global pressure; $\bar{\rho}$ is defined as

$$
\bar{\rho}=\frac{\sum_{\alpha=1}^{n_{p}} \rho_{\alpha}^{2} k_{\alpha}}{\sum_{\alpha=1}^{n_{p}} \rho_{\alpha} k_{\alpha}}
$$

and

$$
k_{\alpha}=\frac{k_{\mathrm{r} \alpha}}{\mu_{\alpha}}
$$

is the mobility of phase $\alpha$, where $k_{\mathrm{r} \alpha}$ is the relative permeability of phase $\alpha$, and $\mu_{\alpha}$ is the dynamics viscosity of phase $\alpha$. Corey's curve is adopted for the relative permeability [13]

$$
\begin{aligned}
k_{\mathrm{rl}} & =\hat{S}^{4} \\
k_{\mathrm{rg}} & =(1-\hat{S})^{2}\left(1-\hat{S}^{2}\right) \quad \text { with } \\
\hat{S} & =\frac{S_{\mathrm{w}}-S_{\mathrm{lr}}}{1-S_{\mathrm{lr}}-S_{\mathrm{gr}}}
\end{aligned}
$$

where $S_{\mathrm{lr}}$ is the residual saturation for the liquid (water); $S_{\mathrm{gr}}$ the residual saturation for the gas (air); and $\hat{S}$ the normalized saturation.

The mass flux of phase $\alpha$ in Equation (3) can be written as follows according to Darcy's law:

$$
\rho_{\alpha} \overline{\mathbf{q}}_{\alpha}=\frac{\rho_{\alpha} k_{\alpha}}{\sum_{\beta=1}^{n_{p}} \rho_{\beta} k_{\beta}}\left[\mathbf{q}+\mathbf{k} \cdot\left[\sum_{\beta=1, \beta \neq \alpha}^{n_{p}} \rho_{\beta} k_{\beta}\left[\nabla P_{c}^{\alpha \beta}+\left(\rho_{\alpha}-\rho_{\beta}\right) \mathbf{b}\right]\right]\right]
$$

where $P_{c}^{\alpha \beta}=p_{\beta}-p_{\alpha}$ is the capillary pressure between phases $\alpha$ and $\beta$. In the present study, the capillary pressure between the phases is ignored, that is, $P_{c}^{\alpha \beta}=0$. As a consequence, the pressure for the different phases $p_{\alpha}$ is all equal to the global pressure $P$.

A finite element Galerkin/finite volume analysis programme DYNAFLOW is used for the simulations [14]. The fully coupled equations of displacements $\left(\mathbf{u}^{\mathrm{s}}\right)$ and pressure $(p)$ are solved simultaneously with a stabilized Galerkin finite element method. The saturation equations are solved using vertex-centered finite volume method with an upwinding scheme and are coupled to pressure and displacements with a staggered approach with iterations.

There are three physical processes taking place in an unsaturated porous medium under external applied loading: (i) pore fluid (water and air) pressure diffusion; (ii) solid skeleton consolidation; 
Table I. Time scales for unsaturated flows in deformable porous media.

\begin{tabular}{ll}
\hline Physical process & \multicolumn{1}{c}{ Time scale } \\
\hline Pore pressure diffusion & $T_{c p}=L^{2} \phi \mu_{\alpha} C_{\mathrm{f}} / k$ \\
Soil consolidation & $T_{c c}=L^{2} \mu_{\alpha} C_{\mathrm{m}} / k$ \\
Saturation front propagation & $T_{c s}=\phi \mu_{x} L_{\mathrm{s}} L /(6 k \Delta p)$ \\
Wave loading & $T_{c w}=\sqrt{2 l} /(\tan \theta \sqrt{g})$ \\
\hline
\end{tabular}

( $\mu_{\alpha}$ is the viscosity of phase $\alpha$, that is, water or air; $C_{\mathrm{f}}$ is the compressibility of pore fluid; $\mu_{x}$ is a fictitious viscosity between $\mu_{\mathrm{a}}$ and $\mu_{\mathrm{w}} ; \phi$ the porosity; $L$ the soil column depth; $k$ the permeability; $C_{\mathrm{m}}$ is the compressibility of matrix; $l$ is the wave excursion distance, that is, the horizontal distance between initial shoreline and maximum runup point; $\theta$ the bed slope; $g$ the gravity constant).

and (iii) saturation front propagation. The characteristic time scales of the individual processes considered here, denoted as $T_{c p}, T_{c c}$, and $T_{c s}$, respectively, are obtained from decoupled models based on the mathematical formulation above [15]. The results presented in Reference [15] are represented in Table I, where the characteristic time scale of each process is expressed in terms of soil and pore fluid properties. The time scale of wave loading, $T_{c w}$, acting at a point onshore on a slope is also included in the table. The analysis in Reference [15] suggests that the transient pore pressure and stress responses are dominated by the pore pressure diffusion and skeleton consolidation, while the steady-state responses are dominated by the saturation front propagation.

\section{SCALING ANALYSIS}

Most experiments are designed to preserve geometric similarity whenever possible. In experiments involving gravity waves, inertial and gravitational forces control the wave propagation and transformation. Therefore, the Froude number $(F r=v / \sqrt{g d}$, with $d$ being the water depth), which represents the ratio of inertial forces to gravitational forces, is usually preserved. When preserving Froude number, in a $1 g$ wave basin and in a $\lambda g$ centrifuge facility, the inertial time is scaled according to

$$
\Lambda_{\mathrm{WB}}[t]=\sqrt{\lambda_{\mathrm{WB}}} \text { and } \Lambda_{\mathrm{CF}}[t]=\lambda_{\mathrm{CF}}
$$

respectively, where $\lambda$ is the geometric length scale ratio of the respective models. The operator $\Lambda[\cdot]$ is defined as the ratio between the quantity in the prototype and that in the model

$$
\Lambda[\cdot]=\frac{[\cdot]_{\text {prototype }}}{[\cdot]_{\text {model }}}
$$

and $\Lambda[L] \equiv \lambda$ is the length scale of the experiment, with $L$ being the length of the soil column. As wave basins and centrifuges generally require different scaling relations, the subscripts WB and $\mathrm{CF}$ are used to indicate scaling relations specific for wave basins and centrifuge facilities, respectively, while $\lambda$ and $\Lambda$ without subscripts indicate general scaling relations applicable to both facilities. Note that in a centrifuge model $\Lambda_{\mathrm{CF}}[g]=1 / \lambda_{\mathrm{CF}}$.

Ideally, the characteristic time scales for all the processes should be scaled correctly, which is generally impossible unless $\lambda=1$ (the model and the prototype have the same scale). In order to 
preserve the interactions among the three processes that govern the soil response during external wave loading, the following three dimensionless parameters:

$$
\begin{aligned}
\gamma_{p w} & =\frac{T_{c p}}{T_{c w}}=\frac{\text { pore pressure diffusion time }}{\text { wave loading time }} \\
\gamma_{c p} & =\frac{T_{c c}}{T_{c p}}=\frac{\text { skeleton consolidation time }}{\text { pore pressure diffusion time }} \\
\gamma_{s w} & =\frac{T_{c s}}{T_{c w}}=\frac{\text { saturation propagation time }}{\text { wave loading time }}
\end{aligned}
$$

should be preserved between the model and the prototype, viz,

$$
\begin{aligned}
& \Lambda\left[\gamma_{p w}\right]=1 \\
& \Lambda\left[\gamma_{c p}\right]=1 \\
& \Lambda\left[\gamma_{s w}\right]=1
\end{aligned}
$$

If $\gamma_{p w}$ is much larger or much smaller than 1, which means that pore fluid pressure diffusion occurs at a very different time scale (larger or smaller) than the wave loading, and hence the two processes are not coupled and transient effects related to pore fluid diffusion are not important. If $\gamma_{c p}$ is much larger than 1 , the increases in pore pressure due to squeezing of the soil skeleton occur over a much larger time scale than the pore fluid pressure diffusion, which implies squeezing effect (reduction of pore spaces) dominates over pore pressure diffusion. It is shown in Reference [15] that $\gamma_{c p}=C_{\mathrm{m}} /\left(\phi C_{\mathrm{f}}\right)$, where $C_{\mathrm{m}}$ and $C_{\mathrm{f}}$ are the compressibility of the skeleton and the pore fluid, respectively. If $\gamma_{s w}$ is much larger than 1 , the saturation propagation occurs over a much larger time scale than the wave loading and the coupling between the two processes is not important, which is the case for transient wave loading over a fine sand bed. A steady-state loading case (such as in storm surges), where it is necessary to preserve $\gamma_{s w}$ and the consequence of failing to do so, is investigated in Section 4.2.

Based on the explanations above, we refer to $\gamma_{p w}$ and $\gamma_{c p}$ as normalized pore pressure diffusion time and normalized relative compressibility (between the skeleton and the pore fluid), respectively, which characterize transient bed responses under wave loading. The parameter $\gamma_{s w}$ characterizes the long-term responses driven by saturation propagation. Non-dimensional numbers $\gamma_{s c}=T_{c s} / T_{c c}$ and $\gamma_{s p}=T_{c s} / T_{c p}$ indicate the coupling between saturation propagation and soil consolidation, and that between saturation propagation and pore pressure diffusion, respectively, which are shown to be not important in either transient responses or steady-state cases because the time scales differ by orders of magnitude [15], and thus they are not further discussed.

In this study, it is assumed that all the bed material properties used in wave basin experiments are the same as in the prototype, except that we can change the soil permeability while keeping the skeleton moduli to be the same. This is possible according to Kimura [16]. Compared with the variation of permeability (one or several orders of magnitude), the porosity and modulus of sand vary in a much smaller scale $(\sim 0.3-0.45)$, and thus it can be considered almost constant for the purpose of scaling analysis. The practical considerations related to these assumptions are discussed in Section 5.1. For centrifuge experiments, the same soil as in the prototype is used in the model, but a liquid other than water (such as a polymer fluid) can be used to obtain different 
viscosity. The air properties, however, may not be scaled since using a different gas would require sealing the bucket containing the soil sample, which would further increase the difficulty of the experiments.

With the assumptions above, for wave basin experiments, Equation (18) is satisfied by choosing soil permeability such that

$$
\Lambda_{\mathrm{WB}}[k]=\lambda_{\mathrm{WB}}^{3 / 2} \quad \text { or } \quad k_{\mathrm{WB}}=\lambda_{\mathrm{WB}}^{-3 / 2} k_{\text {prototype }}
$$

while Equation (19) can be satisfied by using the same fluids (water and air) and using soil with the same compressibility $\left(C_{\mathrm{m}}\right)$ as in the prototype. Parameter $\gamma_{s w}$ (characterizing steadystate/saturation responses) cannot be preserved simultaneously with $\gamma_{p w}$ and $\gamma_{c p}$ (characterizing transient/pore pressure responses) in wave basin experiments, and hence requires a different scaling relation.

For centrifuge experiments, Equation (18) can be satisfied by using the same soil as in the prototype and by using a polymer fluid with viscosity $\lambda_{\mathrm{CF}} \mu_{\mathrm{w}}$, that is,

$$
\Lambda_{\mathrm{CF}}[\mu]=1 / \lambda_{\mathrm{CF}} \quad \text { or } \quad\left[\mu_{\mathrm{w}}\right]_{\mathrm{CF}}=\lambda_{\mathrm{CF}}\left[\mu_{\mathrm{w}}\right]_{\text {prototype }}
$$

where $\left[\mu_{\mathrm{w}}\right]_{\mathrm{CF}}$ is the viscosity of the polymer fluid used in the centrifuge experiment. Equation (19) is satisfied if the liquid used in the centrifuge has the same compressibility as water, which is approximately true. In contrast to the wave basin experiments, parameter $\gamma_{s w}$ can be preserved simultaneously as $\gamma_{p w}$ and $\gamma_{c p}$ in a centrifuge (i.e. Equations (18), (19), and (20) can be satisfied simultaneously) if the liquid density is the same as that of water, which is also approximately true.

When the experiments are scaled to preserve Froude number and transient bed responses via permeability scaling for wave basins and viscosity scaling for centrifuges, that is, according to the scaling requirements in Equations (21) and (22), the scaling relations for the non-dimensional parameters are

$$
\begin{array}{ccc}
\Lambda_{\mathrm{WB}}\left[\gamma_{p w}\right]=1, & \Lambda_{\mathrm{WB}}\left[\gamma_{c p}\right]=1, & \Lambda_{\mathrm{WB}}\left[\gamma_{s w}\right]=\lambda_{\mathrm{WB}} \\
\Lambda_{\mathrm{CF}}\left[\gamma_{p w}\right]=1, & \Lambda_{\mathrm{CF}}\left[\gamma_{c p}\right]=1, & \Lambda_{\mathrm{CF}}\left[\gamma_{s w}\right]=1
\end{array}
$$

for wave basins and centrifuges, respectively. Details of the derivations are presented in Appendix A. Note that air viscosity is not scaled in centrifuge experiments, the consequences of which will be investigated via numerical simulations in Section 4.1 and further discussed in Section 5.2.

A complication in the scaling is the nonlinear constitutive relation of the soil. The moduli of the sand depend on the confining stress, which is typical for noncohesive granular materials. Numerically this behavior can be described by the following models [17]:

$$
\frac{G}{G_{0}}=\left(\frac{\sigma}{\sigma_{0}}\right)^{n} \text { and } \quad \frac{K}{K_{0}}=\left(\frac{\sigma}{\sigma_{0}}\right)^{n}
$$

where $G$ is the shear modulus, $\sigma=\operatorname{tr}\left(\sigma^{\mathrm{s}}\right) / 3$ is the mean effective stress of the skeleton, and the subscript 0 here indicates the values at a reference location, and $n$ is the exponent approximately ranging from 0.2 to 1 according to experimental data available in the literature [18]. In this study, $n=0.5$ is used. Physically, Equation (25) means that the granular soil stiffens with increasing confining stress, as opposed to constant stiffness for materials described by linear elastic models. Depending on whether the loading and unloading moduli are the same, the dependence of skeleton moduli on confining stress is referred to as hypo-elastic or hypo-plastic. The stress-strain relation 


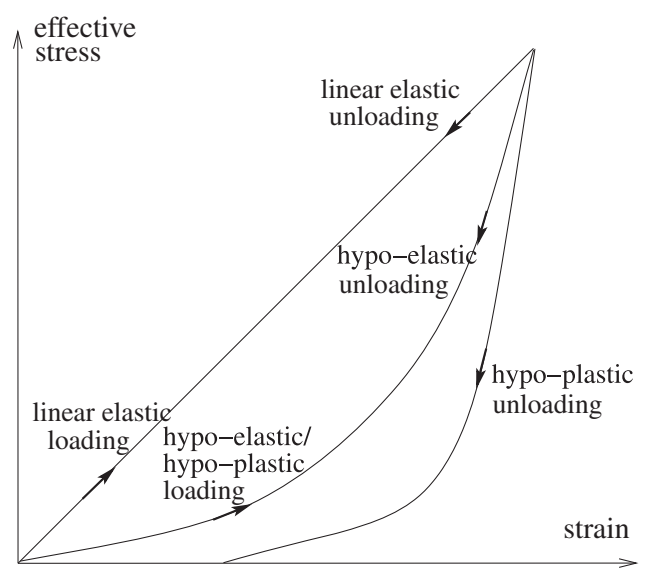

Figure 1. Stress-strain relation during loading and unloading for linear elastic, hypo-elastic, and hypo-plastic constitutive models.

Table II. Physical parameters for the prototype model.

\begin{tabular}{lc}
\hline Parameter & Quantity and unit \\
\hline Gravity constant $(g)$ & $10\left(\mathrm{~m} / \mathrm{s}^{2}\right)$ \\
Young's modulus of skeleton $(E)$ & $1.5 \times 10^{8}(\mathrm{~Pa})$ \\
Poisson's ratio of skeleton $(v)$ & 0.2 \\
Density of soil grains $\left(\rho_{\mathrm{s}}\right)$ & $2650\left(\mathrm{~kg} / \mathrm{m}^{3}\right)$ \\
Porosity of soil $(\phi)$ & 0.4 \\
Permeability $(k)$ & $1.5 \times 10^{-11} \mathrm{~m}^{2}$ \\
Compressibility of water $\left(C_{\mathrm{w}}\right)$ & $4.6 \times 10^{-10}\left(\mathrm{~m}^{2} / \mathrm{N}\right)$ \\
Compressibility of air $\left(C_{\mathrm{a}}\right)$ & $1.0 \times 10^{-5}\left(\mathrm{~m}^{2} / \mathrm{N}\right)$ \\
Density of water $\left(\rho_{\mathrm{w}}\right)$ & $1.0 \times 10^{3}\left(\mathrm{~kg} / \mathrm{m}^{3}\right)$ \\
Density of air $\left(\rho_{\mathrm{a}}\right)$ & $1.0\left(\mathrm{~kg} / \mathrm{m}^{3}\right)$ \\
Viscosity of water $\left(\mu_{\mathrm{w}}\right)$ & $1.0 \times 10^{-3}(\mathrm{~kg} \mathrm{~m} / \mathrm{s})$ \\
Viscosity of air $\left(\mu_{\mathrm{a}}\right)$ & $1.8 \times 10^{-5}(\mathrm{~kg} \mathrm{~m} / \mathrm{s})$ \\
Water residual saturation $\left(S_{\mathrm{lr}}\right)$ & $3 \%$ \\
Air residual saturation $\left(S_{\mathrm{gr}}\right)$ & $3 \%$ \\
\hline
\end{tabular}

during loading and unloading is shown in Figure 1 for linear elastic, hypo-elastic, and hypo-plastic constitutive models.

For linear elastic soils in this study, the elastic moduli (Young's modulus and Poisson's ratio) are as shown in Table II. The shear and bulk moduli are obtained from relations $K=E /[3(1-2 v)]$ and $G=E /[2(1+v)]$, respectively. For the hypo-elastic soil model used in this study, the elastic moduli in the table are the reference values with confining stress $\sigma_{0}=0.165 \mathrm{MPa}$, corresponding to the overburden pressure of $10 \mathrm{~m}$ dry soil. The hypo-plastic soil model uses the same shear and bulk moduli as the hypo-elastic model during the loading phase, while the unloading moduli are twice that of the loading moduli. 


\section{NUMERICAL SIMULATIONS OF SCALING EFFECTS}

To validate the scaling analysis in Section 3, an external wave loading on a soil column is studied. The prototype and two scaled models, one in a wave basin and the other in a centrifuge, are studied. The prototype is a soil column located in a constant 1:15 slope beach, $30 \mathrm{~m}$ onshore from the shoreline, with a saturated zone of $18 \mathrm{~m}$ below the water table and an unsaturated zone of $2 \mathrm{~m}$ above (To have better readability for general audience, the rigorous terminology is not used here. The 'saturated zone' and 'unsaturated zone' here refer to the region below and above the initial water table, respectively. They are generally referred to as phreatic and vadose zones, respectively, in water resource literature.). A schematic is shown in Figure 2. The physical parameters of the soil column are presented in Table II.

Wave loadings caused by both tsunami and hurricane storm surges are studied. The tsunami wave loading applied on the soil column in the prototype is obtained by simulating a solitary wave with an initial offshore height of $12 \mathrm{~m}$ over a water depth of $20 \mathrm{~m}$ running onto the beach (see Figure 2(a)). The water height at the soil column location is recorded. The simulation is based on a depth-averaged numerical model solving nonlinear shallow water equations and Boussinesq equations [19]. The time scale of the tsunami wave loading on the soil column is about $100 \mathrm{~s}$, while for typical hurricane storm surge loading it is of the order of one to several days $\left(10^{5} \mathrm{~s}\right)$. The time series for hurricane loading are obtained by scaling the time of the tsunami loading by a factor of 1000. This is justified by the observation of field measurements of water height time-series onshore during previous hurricanes, which exhibited similar triangular pattern as that from the tsunami simulation that we conducted, but with longer duration and smaller

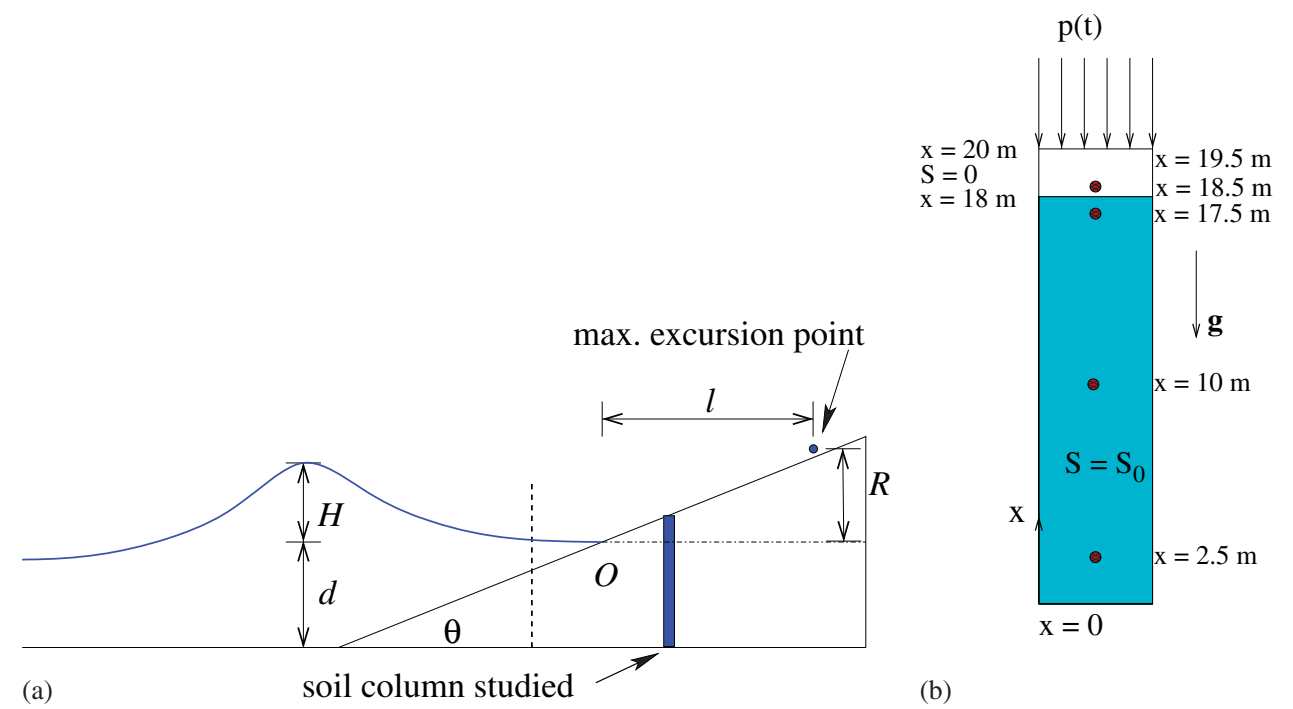

Figure 2. Setup of the problem and the details of the prototype soil column: (a) schematic of wave running up on a sloping beach and the location of the soil column of concern in this study. The vertical dash line roughly divides the bed to the offshore region (left) and near-shore/onshore region (right) and (b) dimensions of the soil column and the locations of 'virtual sensors' where the response time series are studied. 


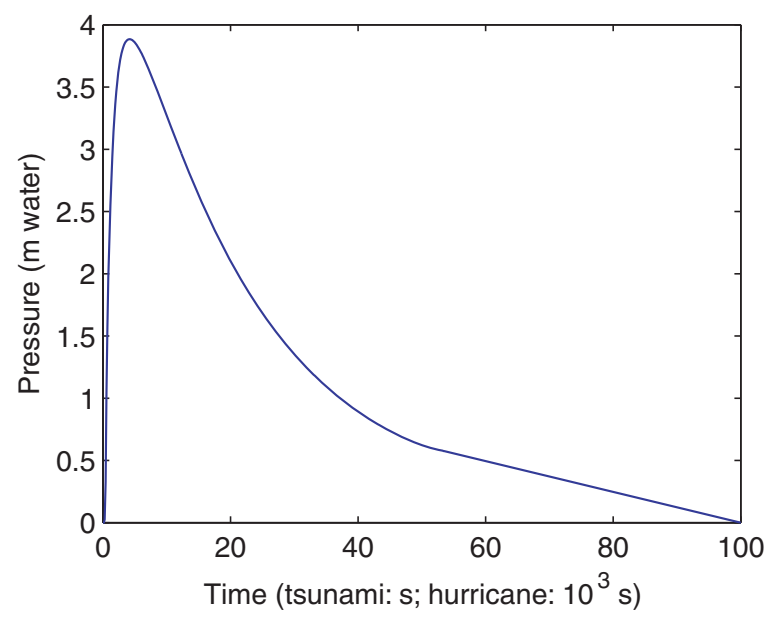

Figure 3. Time series of the prototype wave pressure at the surface of the soil column induced by tsunami and hurricane storm surge.

peak water depth [20]. The time series obtained for tsunami and hurricane loadings are shown in Figure 3.

A $1 g$ wave basin model with a scale ratio of $\lambda_{\mathrm{WB}}=10$, and a $50 \mathrm{~g}$ centrifuge model with a scale ratio of $\lambda_{\mathrm{CF}}=50$ are considered. Geometric similarity is assumed. The scaling is as required to preserve Froude number similarity for the wave $(\Lambda[F r]=1)$ and transient bed response similarities for the soil $\left(\Lambda\left[\gamma_{p w}\right]=\Lambda\left[\gamma_{c p}\right]=1\right)$. The wave loading on the models is obtained according to geometric and Froude number similarity by scaling both the time and the wave height. According to this scaling relation, the loading and response time scales in the prototype and those in the models are shown in Table IV. It can be seen that the tsunami wave loading time scale in the centrifuge is $2 \mathrm{~s}$. For the hurricane storm surge loading, the time scales for the wave basin and centrifuge tests are $3200 \mathrm{~s}$ and $200 \mathrm{~s}$, respectively. The response time scales in the prototype and in the models have a wide range of distribution. The saturation propagation is much slower than other processes and its coupling with those processes is unlikely to be important, which explains why the non-dimensional parameters $\gamma_{s c}=T_{c s} / T_{c c}$ and $\gamma_{s p}=T_{c s} / T_{c p}$ are not important for transient responses. In the prototype, the saturation propagation time scale is much larger than that of the tsunami wave loading, but smaller than that of hurricane loading. This is also true in the centrifuge model, but not in the wave basin model. This is because the saturation propagation is not modeled properly in a wave basin when the experiment is scaled to preserve the transient bed responses. It is illustrated in Equation (23) that the non-dimensional parameter $\gamma_{s w}$ is not preserved in a wave basin. As shown in Table III, the rate of saturation propagation relative to the rate of external wave loading is $\frac{1}{10}$ of the value in the prototype. In a centrifuge, however, the saturation propagation speed is scaled correctly according to Equation (24).

To confirm the observations and the analysis above, a series of numerical simulations are conducted for the prototype and the scaled models to investigate the scaling effects. In all the simulations below, the initial water saturation is assumed to be $S_{\mathrm{W}}=95 \%$ and $S_{\mathrm{W}}=0 \%$ in the region below and above, respectively, in the initial water table. The initial pressure is atmospheric 
Table III. The non-dimensional numbers in wave basin and centrifuge models when scaled to preserve transient response similarity $\left(\gamma_{p w}\right)$.

\begin{tabular}{lcc}
\hline Parameters & Wave basin & Centrifuge \\
\hline$\Lambda\left[\gamma_{p w}\right]$ & 1 & 1 \\
$\Lambda\left[\gamma_{s w}\right]$ & 10 & 1 \\
\hline
\end{tabular}

$\Lambda[\cdot] \equiv[\cdot]_{\text {prototype }} /[\cdot]_{\text {model }}$.

Table IV. The characteristic time scales (in seconds) for various processes including wave loading and soil responses.

\begin{tabular}{lcccc}
\hline Processes & Prototype & Wave basin & Centrifuge & Unit \\
\hline Tsunami loading & 100 & 32 & 2 & $\mathrm{~s}$ \\
Hurricane loading & $10^{4}$ & 3200 & 200 & $\mathrm{~s}$ \\
Saturation propagation $\left(T_{c s}\right)$ & 4500 & $1.4 \times 10^{4}$ & 90 & $\mathrm{~s}$ \\
Air pressure diffusion $\left(T_{c p, \mathrm{a}}\right)$ & 200 & 63.2 & $8.0 \times 10^{-2}$ & $\mathrm{~s}$ \\
Water pressure diffusion $\left(T_{c p, \mathrm{w}}\right)$ & 10 & 3.2 & 0.20 & $\mathrm{~s}$ \\
Skeleton consolidation $\left(T_{c c}\right)$ & 160 & 16 & 3.2 & $\mathrm{~s}$ \\
\hline
\end{tabular}

$\left(1 \times 10^{5} \mathrm{~Pa}\right)$ at the top surface and the initial pressure distribution is hydrostatic elsewhere in the soil column domain. Body force (gravity) is considered. The top boundary is subject to the surface water pressure resulting from the wave loading, while the effective stress is zero. The pressure at the bottom boundary is given a prescribed value such that the initial hydrostatic pressure in the soil column is balanced exactly. The bottom has no displacements. The water saturation is $100 \%$ at the top boundary whenever there is an external pressure caused by surface waves, and is $0 \%$ otherwise. The water saturation is fixed at $95 \%$ at the bottom boundary. All simulations are conducted with 200 two-node linear elements with a uniform size of $\Delta x=L / 200(L=20 \mathrm{~m})$. Time step size $(\Delta t)$ is determined according to the characteristic time of each problem. For the prototype, it is chosen $\Delta t=0.05 \mathrm{~s}$ for tsunami loading and $\Delta t=1 \mathrm{~s}$ for hurricane storm surge loading. In scaled models, the time steps sizes, $\Delta t$, are scaled accordingly.

If the soil were linear elastic, the permeability scaling according to Equation (21) would yield perfectly scaled pore pressure and stress responses in a wave basin model under transient loading (when $\gamma_{s w} \gg 1$ ). However, similitude of the displacement responses is not satisfied with the scaling relation in Equation (21); the displacements in the model should thus be approximately $\frac{1}{10}$ of their correctly scaled values. These inferences from the scaling analysis have been confirmed by our numerical simulations, which also confirmed that the transient responses are indeed controlled by the non-dimensional parameters $\gamma_{p w}$ and $\gamma_{c p}$. However, for transient loadings, these simulation are only meaningful when the soil column of concern is very shallow and thus the hypo-plasticity is not important. As we are more interested in realistic soil behaviors, these idealized validation examples are not shown here. Instead, results obtained using more realistic hypo-plastic models are shown below. 

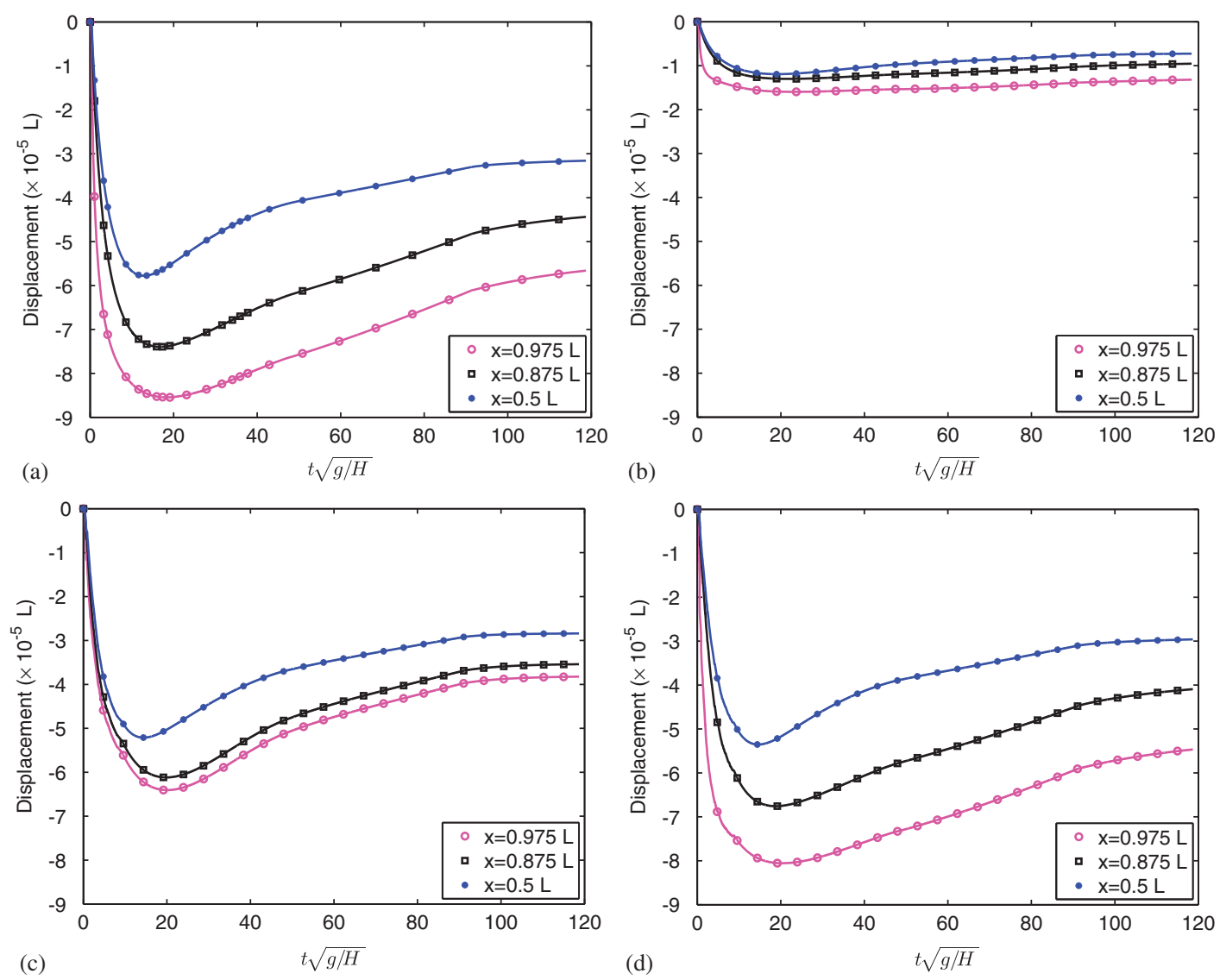

Figure 4. Effects of hypo-plasticity and air viscosity scaling on the displacements. The displacement responses under tsunami wave loading, with hypo-plastic soil model, for: (a) the prototype; (b) the wave basin model with scaled permeability; (c) the centrifuge model with scaled water viscosity; and (d) the centrifuge model assuming the viscosity of both the water and the air are scaled.

\subsection{Effects of hypo-plasticity and air viscosity}

To illustrate the effects of the dependence of soil skeleton moduli on the confining stress, the tsunami wave runup case as described above and illustrated in Figure 2(a) is simulated with a hypoplastic soil model. The predicted displacement responses for the prototype and the scaled models are presented in Figure 4. Displacements are shown because the displacement is more directly influenced by the hypo-plasticity of the soils compared with the pressures. The displacement responses in the wave basin model (Figure 4(b)) are significantly different from those in the prototype (Figure 4(a)), in terms of both magnitude and general trend. The differences in magnitude are due to the improper scaling of soil skeleton moduli. The difference in the trend is due to nonlinear soil responses resulting from the hypo-plasticity.

The responses from the centrifuge model shown in Figure 4(c) are not completely the same as in the prototype. Although the approximate displacement magnitudes in the model is the same as 
those in the prototype, the trend is slightly different. The discrepancy is probably due to the fact that the viscosity of the air is not properly scaled. To confirm this conjecture, a simulation is conducted assuming that the viscosity of the water and the air is scaled as required. The displacement responses of this simulation are presented in Figure 4(d). It can be seen that the scaled responses in this case are identical to those in the prototype. This set of simulations demonstrates that: (i) the hypo-plastic soil behavior is a challenge for wave basin experiments studying wave-soil interactions if the displacements are important; (ii) centrifuges are able to model the hypo-plastic behavior of soils because the stresses in the model are the same as in the prototype; and (iii) inability to scale the air viscosity in centrifuge experiments is a problem for the study of unsaturated soils, and thus caution must be exercised in the interpretation of the results when unsaturated zones (i.e. zones with significant portion of air content) are present, for example, in the near-shore region. When the soil is fully or nearly fully saturated, the scaling of air viscosity is not a problem. This issue is further discussed in Section 5.2.

\subsection{Effects of loading duration}

The scaling relation for $\gamma_{s w}$ in Table III suggests that in wave basin models scaled to preserve Froude number and transient responses, the steady-state responses are not 'correctly' scaled. In can be seen that the saturation propagation rate relative to the wave loading rate is $\frac{1}{10}$ to that in the prototype. Therefore, when the loading duration is long enough for the saturation propagation to play a role in the system responses, scaling the experiments to preserve the transient responses is not suitable. In a centrifuge, however, the steady-state responses and the transient responses are simultaneously preserved by the same scaling relation $\Lambda_{\mathrm{CF}}[\mu]=1 / \lambda_{\mathrm{CF}}$. The challenge for the centrifuge experiment lies in the difficulty of scaling air viscosity, as mentioned previously. This analysis is validated by a simulation of hurricane storm surge loading (with a duration of $T_{\mathrm{w}}=10^{5} \mathrm{~s}$ ) on the same soil column as in Figure 2. The simulation is conducted from $t=0 \mathrm{~s}$ to $t=1.3 \times 10^{5} \mathrm{~s}$ in the prototype (no wave loading between $1.0 \times 10^{5} \mathrm{~s}$ and $1.3 \times 10^{5} \mathrm{~s}$ ). The time span and wave loading duration in the models are scaled accordingly. The soils are assumed to be linear elastic in this set of simulations because it is found that linear elastic, hypo-elastic, and hypo-plastic simulations gave the same results for pore pressure and stress responses for the long-duration loading considered in this section. This is probably because the pore pressure and stress responses are dominated by the average moduli and are only indirectly influenced by the confining effects (as opposed to displacements). Note that when displacement responses are of concern, linear elastic soil models would be inadequate and hypo-plastic models are needed.

The pressure responses at three points for the prototype and for experimental models with different scaling are presented in Figures 5(a)-(d). The corresponding saturation responses are presented in Figures 6(a)-(d). The time is normalized by wave loading duration $T_{\mathrm{w}}$, and the pressure variation is normalized by the maximum pressure, both in their respective cases. The pressure and saturation responses for the prototype are shown in Figures 5(a) and 6(a), respectively. The pressures at $x=0.975 L$ and $x=0.875 L$ are higher than the applied pressure at $x=L$ for $t>0.4 T_{\mathrm{w}}$. This is caused by the increase in the hydrostatic pressure due to the water penetration into the initially dry region (see Figure 6(a)).

The pressure and saturation responses in a wave basin model are shown in Figures 5(b) and 6(b), respectively, which are qualitatively different from the prototype responses. In Figure 5(b), the pressures at the three points generally follow the trend of the applied pressure at $x=L$. The pressure at $x=0.975 \mathrm{~L}$ rises above that at $x=L$ due to the slight water invasion between $x=0.975 \mathrm{~L}$ 

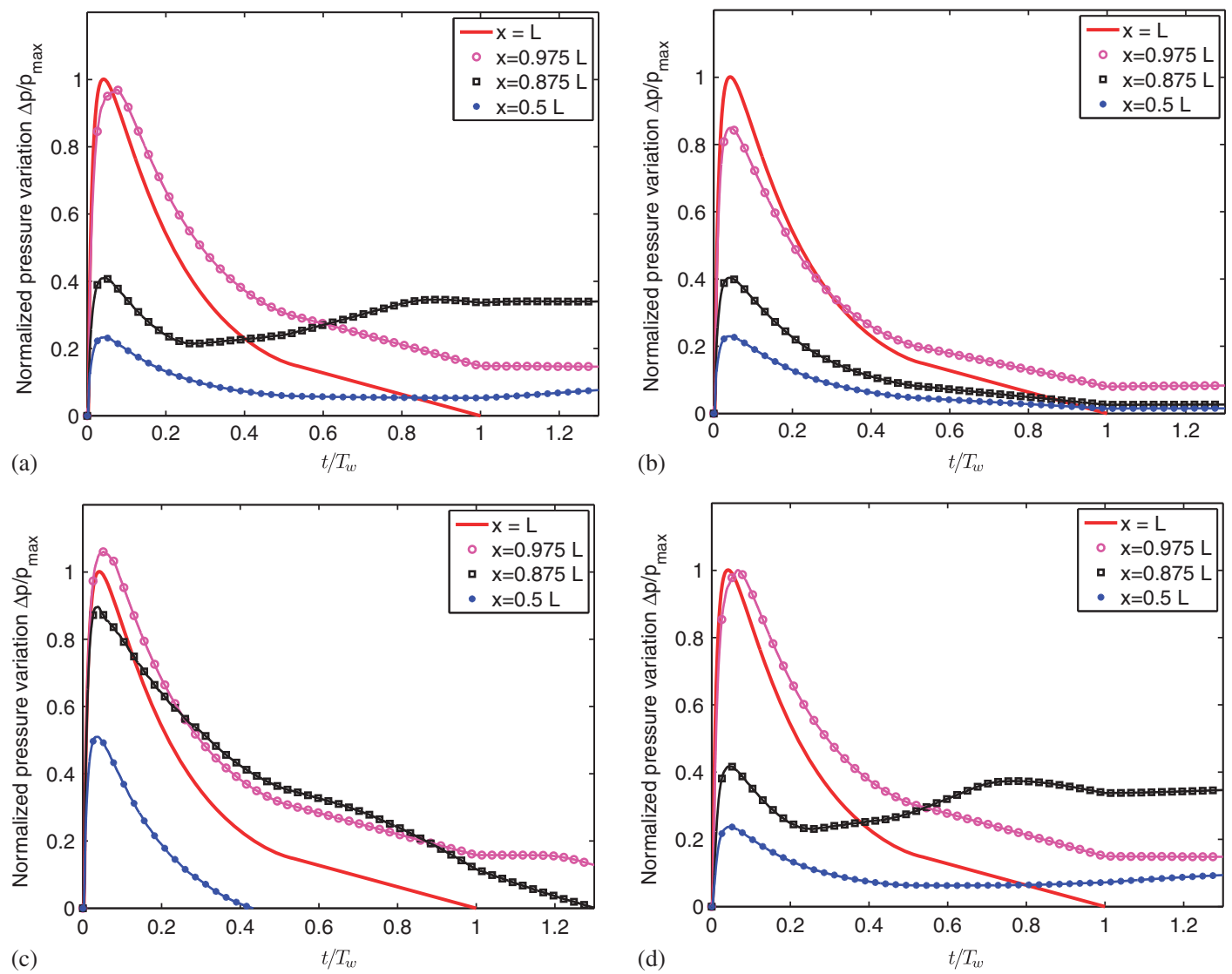

Figure 5. Effects of permeability scaling (wave basin) and viscosity scaling (centrifuge) for loadings of long durations. Pore pressure responses under hurricane wave loading, with linear elastic soil model, for: (a) the prototype; (b) the wave basin model with scaled permeability; (c) the centrifuge model with scaled water viscosity only; and (d) the centrifuge model with scaled water viscosity and scaled air viscosity. The time is normalized by the duration of wave loading $\left(T_{\mathrm{w}}\right)$. The pressure variation is normalized by the maximum loading pressure ( $3.88 \mathrm{~m}$ water head in prototype and scaled accordingly in scaled models).

and $x=L$. In the wave basin model, the water front has not yet reached the location at or below $x=0.975 \mathrm{~L}$ during the simulation time span, which is shown in Figure 6(b). This is because the saturation responses are not correctly scaled in the wave basin. The responses in the centrifuge model, as presented in Figures 5(c) and 6(c) for pressure and saturation, respectively, show much better agreements with those in the prototype compared with the wave basin model. This is because the scaling relation in the centrifuge model in Equation (22) preserves both transient and steady-state responses. Some discrepancies are still observed due to the fact that the air viscosity is not scaled.

To illustrate the effects of the air viscosity scaling, another simulation is conducted assuming the air viscosity to be correctly scaled. The pressure and saturation responses in the centrifuge model under this assumption are presented in Figures 5(d) and 6(d), respectively. Perfectly scaled results are obtained in this simulation, as predicted by the scaling analysis. Therefore, when unsaturated zones are presented in the experiment, failing to scale air viscosity may be a hurdle for obtaining 

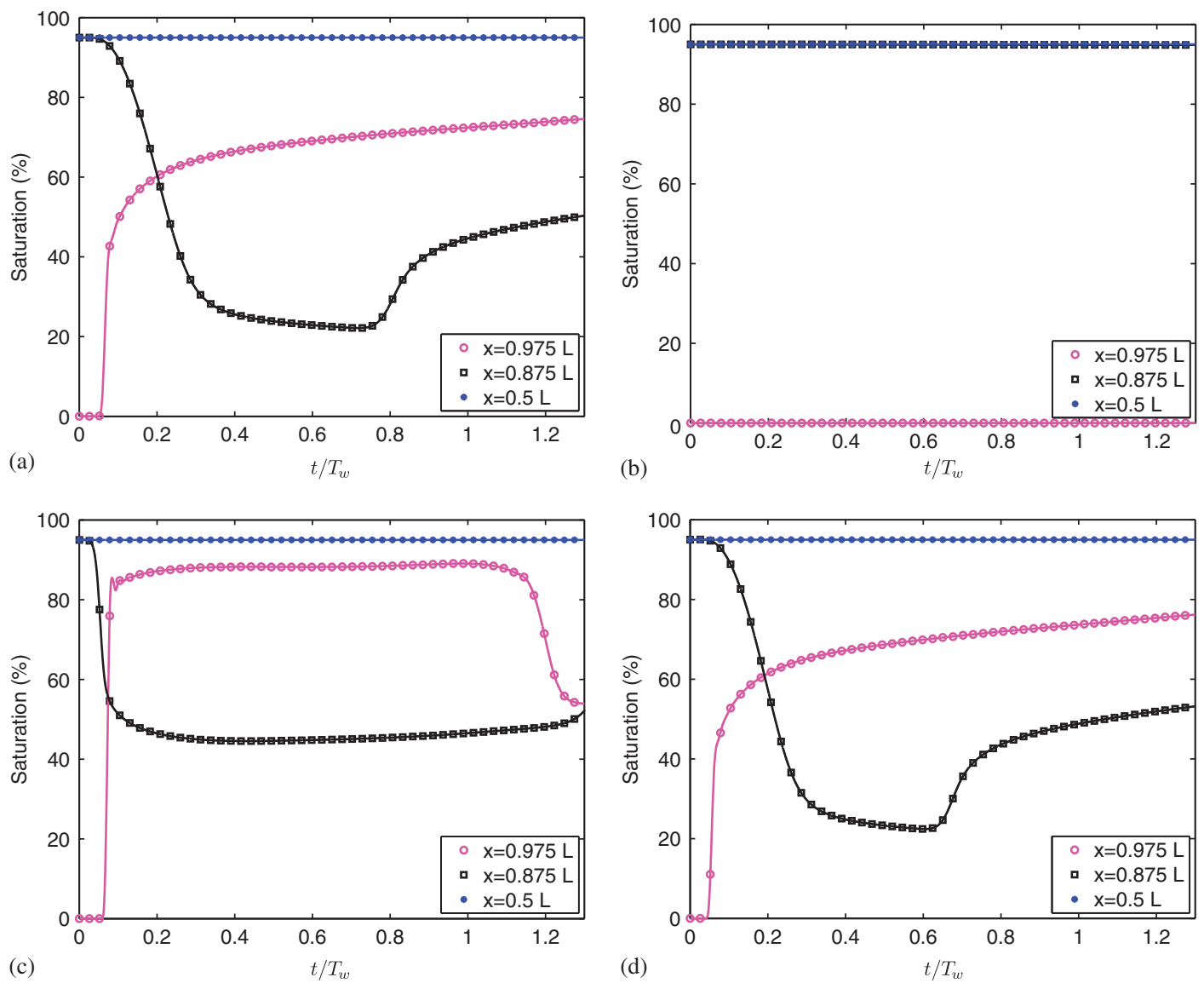

Figure 6. Effects of permeability scaling (wave basin) and viscosity scaling (centrifuge) for wave loadings of long durations. Saturation responses under hurricane wave loading, with linear elastic soil model, for: (a) the prototype; (b) the wave basin model with scaled permeability; (c) the centrifuge model with scaled water viscosity; and (d) the centrifuge model assuming the viscosity of both the water and the air are scaled. The time is normalized by the duration of wave loading $\left(T_{\mathrm{w}}\right)$.

correctly scaled results. In these situations, wave basin experiments should be used with a scaling to preserve steady-state response (saturation propagation), characterized by parameter $\gamma_{s w}$. That is, for saturation-dominated long-term responses, the pressure and saturation responses should be studied in wave basins with scaling relation $\Lambda_{\mathrm{WB}}\left[\gamma_{s w}\right]=1$, which would require $\Lambda_{\mathrm{WB}}[k]=\sqrt{\lambda_{\mathrm{WB}}}$.

\section{DISCUSSION}

\subsection{Scaling of sand in wave basin}

The analysis and simulations shown above are simplified and idealized. In particular, it is assumed that the permeability for the sand used in wave basins can be changed from the prototype without 
modifying other moduli, which may be difficult to achieve in reality. Kimura [16] investigated the dependence of bulk modulus of porous granular materials on grain sizes by measuring acoustic wave speeds in samples of glass beads and marine sediments. The observations suggest that the frame bulk modulus of vacuum-saturated samples (corresponding to the skeleton bulk modulus here) does not vary significantly with grain sizes. The skeleton compressibility depends more on the grain materials than on the grain sizes. Therefore, in a wave basin experiments, the sand can be extracted from the field (prototype) and sieved to separate different grain size groups. The desired grain size groups can be selected to obtain the desired permeability, according to empirical relations between permeability, porosity, and particle diameter such as the Kozeny-Carman formula [21]. As the actual permeability may deviate from the predictions of empirical formulas, some trialand-error laboratory tests may be needed to find the exact grain sizes distribution to achieve the required permeability. If the same materials are used for both the prototype and the model, then the compressibility and other moduli should be similar, if not identical, which should again be confirmed with the laboratory tests.

\subsection{Air viscosity scaling in unsaturated zones}

From the numerical examples shown in Sections 4.1 and 4.2, it is concluded that perfect scaling is not possible when an unsaturated zone is present and the air viscosity is not properly scaled. In Figure 4, the displacements at $x=0.5 L$ (in the saturated zone) are the same whether the air viscosity is scaled (Figure 4(c)) or unscaled (Figure 4(d)). The displacements at $x=0.875 \mathrm{~L}$ and $x=0.975 \mathrm{~L}$, both located in the unsaturated zone, differ depending on whether the air viscosity is scaled (Figure 4(d)) or not (Figure 4(c)). However, this observation cannot be generalized. When the air viscosity is not scaled, the responses in the saturated zone may be affected as well, depending on the loading and what responses are of concern. For example, the pore pressure responses at $x=0.5 \mathrm{~L}$ in Figures 5(c) (unscaled air viscosity) and 5(d) (scaled air viscosity) are different, even though this point is located in the saturated zone. Other displacement and pore pressure responses omitted here also suggest that the responses in saturated zone can be affected by the improper scaling of air viscosity. Therefore, when a centrifuge experiment is conducted with unsaturated soil and the air viscosity is not scaled, numerical simulations should be conducted to examine whether the test results can be extrapolated to prototypes.

\subsection{Recommendations on wave-soil interactions experiments}

According to the scaling analysis and numerical simulations above, to investigate the wave-soil interaction processes, experiments in wave basins and in centrifuge facilities should be combined to study different aspects of the physical processes. Specifically, in terms of pore pressure and effective stress responses (including bed failures), the wave basin results are reliable when the soil column of concern is shallow and thus the hypo-plasticity effects of soil are not significant, while the centrifuges would give better predictions in the saturated region (where air does not play important roles). In terms of displacement responses, the centrifuge results are more reliable when only saturated soils are present. In wave basins the displacement responses are not correctly scaled with the permeability scaling.

Therefore, when the process of transient wave runup/drawdown on a slope is experimentally modeled both in a wave basin and in a centrifuge facility, the recommendations on results interpretation are as follows. In the offshore region (roughly corresponding to the left side of the vertical 
dash line in Figure 2(a)), the pore pressure, stress, and displacement results from the centrifuge experiments can be extrapolated to the prototype with confidence. In the region close to or landward of the shoreline (corresponding to the right side of the vertical dash line in Figure 2(a)), the pore pressure and effective stress in the shallow region (top part of the soil) from the wave basin experiments can be extrapolated to prototype. However, in this region, the stresses deep in the soil column and all the displacements, either from the wave basin or from the centrifuge, should not be extrapolated. If these quantities are important, either numerical simulations should be conducted, or if possible centrifuge experiments should be conducted with sealed bucket and gas with scaled viscosity in order to achieve correct scaling.

In the cases where soil failures could be caused by both transient wave loading and steady-state wave loading, parameters $\gamma_{p w}, \gamma_{c p}$, and $\gamma_{s w}$ all need to be preserved, and thus only centrifuge tests can be used.

\section{CONCLUSION}

The scaling of dynamic wave-soil interaction problems is studied in this work. Non-dimensional parameters governing the transient and steady-state soil responses of a soil column on the beach under tsunami and storm surge loading are analyzed based on simplified models. Scaling relations to preserve transient responses are proposed for both wave basin and centrifuge wave tank facilities. Numerical simulations are conducted to validate the proposed scaling relations and to assess the influences of other factors that may appear in actual experiments. Based on the analysis and simulations, the following conclusion are drawn:

(i) Wave basin experiments are capable of modeling the transient responses under wave loading by permeability scaling when soil hypo-plasticity and saturation propagation are not important. Steady-state responses are not scaled properly in the wave basins when the experiment is scaled to preserve transient responses. The major limitation of $1 g$ wave basin experiments lies in the difficulty in modeling the stress-dependent constitutive behaviors of soils.

(ii) Centrifuge wave test facilities are also capable of scaling the transient responses by scaling the liquid viscosity. They are particularly suitable for the cases when the soils exhibit complex constitutive behaviors since the same soil as in prototype is used, and the stresses are preserved. The limitations of the centrifuge facility include the difficulty in scaling air viscosity.

(iii) Therefore, in the experimental studies of wave-soil interactions concerning the dynamic bed responses such as soil failure and localization, experiments using both wave basin and centrifuge facilities should be combined to study the scaling effects and to understand the different aspects of the physical processes.

\section{APPENDIX A: DERIVATION OF SCALING RELATIONS}

The derivation of the scaling relations in Equations (21)-(24) is presented in detail as follows. 
According to the characteristic time scales presented in Table I, three non-dimensional parameters are defined:

$$
\begin{aligned}
& \gamma_{p w}=\frac{T_{c p}}{T_{c w}}=\frac{L^{2} \phi \mu C_{\mathrm{f}} / k}{\sqrt{2 l} /(\tan \theta \sqrt{g})} \\
& \gamma_{c p}=\frac{T_{c c}}{T_{c p}}=\frac{C_{\mathrm{m}}}{\phi C_{p}} \\
& \gamma_{s w}=\frac{T_{c s}}{T_{c w}}=\frac{\phi \mu L_{\mathrm{s}} L /(6 k \Delta p)}{\sqrt{2 l} /(\tan \theta \sqrt{g})}
\end{aligned}
$$

Refer to the text in Section 2 for the definition of the symbols. Since $\tan \theta$ and the ratios of different lengths are always preserved in the models, the definitions above are modified slightly as follows:

$$
\begin{aligned}
\gamma_{p w} & =\frac{L^{\frac{3}{2}} \phi \mu C_{\mathrm{f}} \sqrt{g}}{k} \\
\gamma_{c p} & =\frac{T_{c c}}{T_{c p}}=\frac{C_{\mathrm{m}}}{\phi C_{p}} \\
\gamma_{s w} & =\frac{\phi \mu \sqrt{L}}{\rho_{\mathrm{f}} k \sqrt{g}}
\end{aligned}
$$

where the pressure difference $\Delta p$ is replaced with $\rho_{\mathrm{f}} g L$. In order to preserve transient responses, we need

$$
\begin{aligned}
& \Lambda\left[\gamma_{p w}\right]=\frac{\lambda^{3 / 2} \Lambda\left[\phi \mu C_{\mathrm{f}} \sqrt{g}\right]}{\Lambda[k]}=1 \\
& \Lambda\left[\gamma_{c p}\right]=\frac{\Lambda\left[C_{\mathrm{m}}\right]}{\Lambda\left[\phi C_{p}\right]}=1
\end{aligned}
$$

In a wave basin, Equation (A4) is achieved by scaling the permeability. Since $\Lambda_{\mathrm{WB}}[\phi]=\Lambda_{\mathrm{WB}}\left[C_{\mathrm{f}}\right]=$ $\Lambda_{\mathrm{WB}}[g]=1$, Equation (A4) leads to

$$
\Lambda_{\mathrm{WB}}[k]=\lambda_{\mathrm{WB}}^{3 / 2}
$$

In a centrifuge facility, Equation (A4) is achieved by changing the fluid viscosity. Since $\Lambda_{\mathrm{CF}}[g]=$ $1 / \lambda_{\mathrm{CF}}$ and $\Lambda_{\mathrm{CF}}[k]=\Lambda_{\mathrm{CF}}[\phi]=1$, assuming $\Lambda_{\mathrm{CF}}\left[C_{\mathrm{f}}\right]=1$, we have

$$
\Lambda_{\mathrm{CF}}[\mu]=\frac{1}{\lambda_{\mathrm{CF}}^{3 / 2} \sqrt{\Lambda_{\mathrm{CF}}[g]}}=\frac{1}{\lambda_{\mathrm{CF}}}
$$

Equations (A6) and (A7) are the scaling relations to preserve transient responses in wave basins and centrifuge facilities, respectively. It is also necessary to satisfy Equation (A2). This is guaranteed if the soils are assumed to be linear elastic. For hypo-elastic or hypo-plastic soils, only centrifuge models can satisfy Equation (A2) as the same soils are used and the stresses are the same in the model as in the prototype. Assuming $C_{\mathrm{m}} \propto \sqrt{\sigma}$, then in wave basin models, $\gamma_{c p}=\sqrt{\lambda_{\mathrm{WB}}}$, suggesting 
the soil in the model is softer (due to lower confining stress) and the squeezing of the skeleton is overrepresented.

Now that we have the scaling relations required to preserve Froude number and transient responses (i.e. Equation (A6) for wave basins and Equation (A7) for centrifuges), it is interesting to see how the other non-dimensional number $\gamma_{s w}$ is scaled in this set of scaling relations.

For wave basin models, the non-dimensional parameter governing the steady-state responses is scaled as follows:

$$
\Lambda_{\mathrm{WB}}\left[\gamma_{s w}\right]=\frac{\Lambda_{\mathrm{WB}}[\mu] \sqrt{\lambda_{\mathrm{WB}}}}{\Lambda_{\mathrm{WB}}\left[\rho_{\mathrm{f}}\right] \Lambda_{\mathrm{WB}}[k] \sqrt{\Lambda_{\mathrm{WB}}[g]}}=1 / \lambda_{\mathrm{WB}}
$$

since $\Lambda_{\mathrm{WB}}[\mu]=\Lambda_{\mathrm{WB}}\left[\rho_{\mathrm{f}}\right]=\Lambda_{\mathrm{WB}}[g]=1, \Lambda_{\mathrm{WB}}[k]=\lambda_{\mathrm{WB}}^{3 / 2}$, and assuming $\Lambda_{\mathrm{WB}}[\phi] \approx 1$, suggesting that the saturation propagation speed relative to the wave speed in the wave basin model is $1 / \lambda_{\mathrm{WB}}$ of (and thus slower than) that in the prototype. For centrifuge, the scaling for $\gamma_{s w}$ is:

$$
\Lambda_{\mathrm{CF}}\left[\gamma_{s w}\right]=\frac{\Lambda_{\mathrm{CF}}[\mu] \sqrt{\lambda_{\mathrm{CF}}}}{\Lambda_{\mathrm{CF}}\left[\rho_{\mathrm{f}}\right] \Lambda_{\mathrm{CF}}[k] \sqrt{\Lambda_{\mathrm{CF}}[g]}}=1
$$

since $\Lambda_{\mathrm{CF}}\left[\rho_{\mathrm{f}}\right] \approx 1, \Lambda_{\mathrm{CF}}[k]=1, \Lambda_{\mathrm{CF}}[\mu]=1 / \lambda_{\mathrm{CF}}$, and $\Lambda_{\mathrm{CF}}[g]=1 / \lambda_{\mathrm{CF}}$.

\section{ACKNOWLEDGEMENTS}

The authors would like to acknowledge funding by the National Science Foundation through grant no. 0649155 and no. 0530759.

\section{REFERENCES}

1. Biot M. General theory of three-dimensional consolidation. Journal of Applied Physics 1941; 12(2):155-164.

2. Detournay E, Cheng AHD. Fundamentals of poroelasticity. In Comprehensive Rock Engineering: Principles, Practice and Projects, Chapter 5, Fairhurst C (ed.), vol. 2. Pergamon: New York, 1993.

3. Coussy O. Poromechanics. Wiley: Hoboken, NJ, 2004.

4. Zienkiewicz O, Shiomi T. Dynamic behaviour of saturated porous media: the generalized Biot formulation and its numerical solution. International Journal for Numerical and Analytical Methods in Geomechanics 1984; 8:71-96.

5. Prévost JH. Nonlinear transient phenomena in saturated porous media. Computer Methods in Applied Mechanics 1982; 30(1):3-18.

6. Schrefler B, Scotta R. A fully coupled dynamic model for two-phase fluid flow in deformable porous media. Computer Methods in Applied Mechanics 2001; 190:3223-3246.

7. Kobayashi N, Lawrence A. Cross-shore sediment transport under breaking solitary waves. Journal of Geophysical Research 2004; 109:C030047.

8. Xiao H, Young YL, Maddux T. Runup and drawdown of breaking solitary waves over a fine sand beach. Part I: experimental investigation. Marine Geology 2009; under review.

9. Coe C, Prévost J, Scanlan R. Dynamic stress wave reflections/attenuation: earthquake simulation in centrifuge soil models. Earthquake Engineering and Structural Dynamics 1985; 13:109-128.

10. Walsh J, Charlie W. Stress wave propagation in unsaturated sands-modeling. Technical Report, Colorado State University, Fort Collins, CO, 1993.

11. Sassa S, Sekiguchi H. Wave-induced liquefaction of beds of sand in a centrifuge. Geotechnique 1999; 49(5): 621-638.

12. Sassa S, Sekiguchi H. Analysis of wave-induced liquefaction of sand beds. Geotechnique 2001; 51(2):115-126.

13. Corey AT. The interrelation between gas and oil relative permeabilities. Producers Monthly 1954; 19(1):38-41.

14. Prévost JH. DYNAFLOW: A Nonlinear Transient Finite Element Analysis Programme, Department of Civil Engineering, Princeton University, Princeton, NJ, 1983. (Last updated 2008.) 
15. Xiao H, Young YL, Prévost JH. Multiphase flow in deformable porous media under extreme wave loading. 2009; under review.

16. Kimura M. Frame bulk modulus of porous granular marine sediments. Journal of Acoustical Society of America 2006; 120(2):699-710.

17. Richart FE, Hall JR, Woods RD. Vibrations of Soils and Foundations. Prentice-Hall: Englewood Cliffs, NJ, 1970.

18. Kazachenko LS, Kulinich YV. Mechanical properties of sand under pressure. Journal of Mining Science 1972; 8(1):19-26.

19. Xiao H, Young YL, Prévost JH. Runup and drawdown of breaking solitary waves over a fine sand beach. Part II: numerical simulation. Marine Geology 2009; under review.

20. McGee BD, Goree BB, Tollett RW, Woodward BK, Kress WH. Hurricane Rita surge data, southwestern Louisiana and southeastern Texas, September-November 2005. Online Document. Available from: http://pubs.usgs.gov/ds/2006/220/.

21. Costa A. Permeability-porosity relationship: a reexamination of the Kozeny-Carman equation based on a fractal pore-space geometry assumption. Geophysical Research Letters 2006; 33:L02318. 